\title{
PPARס: a dagger in the heart of the metabolic syndrome
}

\author{
Grant D. Barish, ${ }^{1,2}$ Vihang A. Narkar, ${ }^{1}$ and Ronald M. Evans ${ }^{1}$
}

${ }^{1}$ Howard Hughes Medical Institute, Gene Expression Laboratory, Salk Institute for Biological Studies, La Jolla, California, USA. 2Division of Endocrinology and Metabolism, Department of Medicine, UCSF, San Francisco, California, USA.

\begin{abstract}
Obesity is a growing threat to global health by virtue of its association with insulin resistance, glucose intolerance, hypertension, and dyslipidemia, collectively known as the metabolic syndrome or syndrome $X$. The nuclear receptors PPAR $\alpha$ and PPAR $\gamma$ are therapeutic targets for hypertriglyceridemia and insulin resistance, respectively, and drugs that modulate these receptors are currently in clinical use. More recent work on the less-described PPAR isotype PPAR $\delta$ has uncovered a dual benefit for both hypertriglyceridemia and insulin resistance, highlighting the broad potential of PPAR $\delta$ in the treatment of metabolic disease. PPAR $\delta$ enhances fatty acid catabolism and energy uncoupling in adipose tissue and muscle, and it suppresses macrophage-derived inflammation. Its combined activities in these and other tissues make it a multifaceted therapeutic target for the metabolic syndrome with the potential to control weight gain, enhance physical endurance, improve insulin sensitivity, and ameliorate atherosclerosis.
\end{abstract}

\section{Introduction}

The prevalence of adult obesity has increased an alarming $75 \%$ since 1980 , rendering a third of men and women obese in the US (1). This unabated rise has spawned proportionate increases in obesity-associated metabolic disorders, including glucose intolerance, insulin resistance, dyslipidemia, and hypertension, that are well-established risk factors for cardiovascular disease. Known as the metabolic syndrome or syndrome $\mathrm{X}$, this dangerous cluster of pathologies accounts for $6-7 \%$ of all-cause mortality and is an expanding health threat. In fact, it is predicted that life expectancy will plateau or decline in the US within the first half of this century because of the magnitude of obesity-associated conditions and the increased rates of obesity in younger populations, particularly children $(2,3)$. The global obesity problem will require complex solutions, including public health efforts to diminish portion sizes, improve food choices, increase physical activity levels, and raise public awareness. In addition to social and behavioral changes, however, pharmacological interventions to diminish diabetic and cardiovascular complications of the metabolic syndrome are urgently needed.

The pathophysiology underlying the metabolic syndrome is incompletely understood, but insulin resistance appears to be an important component $(4,5)$. Insulin resistance is marked by hyperinsulinemia, enhanced hepatic gluconeogenesis, and impaired insulin-stimulated glucose uptake into skeletal muscle and fat. Elevated levels of circulating FFAs, associated with obesity and insulin resistance, increase fat accumulation in insulin target tissues and contribute to defective insulin action. Indeed, intramuscular fat, based on NMR spectroscopy, correlates strongly with insulin resistance (6). Obese adipose tissue-derived inflammation and altered adipokine secretion may also inhibit insulin signals and affect systemic metabolism (7). The resulting hyperglycemia, dyslipidemia, and hypertension of the metabolic syndrome cause endothelial dysfunction and hasten atherogenesis. Mono-

Nonstandard abbreviations used: APC, adenomatous polyposis coli; BCL-6, B cell lymphoma-6; DR, direct repeat; MCP-1, monocyte chemoattractant protein-1; RXR, retinoid X receptor; UCP, uncoupling protein; VP16, viral protein 16.

Conflict of interest: The authors have declared that no conflict of interest exists. Citation for this article: J. Clin. Invest. 116:590-597 (2006). doi:10.1172/JCI27955. cyte-derived macrophages are recruited to the vascular wall, where uptake of modified LDL cholesterol drives their development into so-called foam cells. These cholesterol-loaded macrophages elaborate inflammatory mediators and matrix remodeling enzymes, begetting further inflammation and the formation of complex atherosclerotic lesions through interactions and recruitment of vascular cells, $\mathrm{T}$ cells, and additional macrophages (8). Ultimately, such changes put metabolic syndrome patients at high risk for heart attack and stroke (9).

\section{PPARs: lipid sensors and transcriptional switches}

The identification of PPARs as molecular targets for drugs to treat hypertriglyceridemia and type 2 diabetes mellitus has fueled interest in their biology and potential as targets to treat the metabolic syndrome. PPARs are members of the nuclear receptor superfamily of ligand-inducible transcription factors. They form heterodimers with retinoid X receptors (RXRs) and bind to consensus DNA sites composed of direct repeats (DRs) of hexameric DNA sequences separated by $1 \mathrm{bp}$ (DR1). In the absence of ligand, PPAR-RXR heterodimers recruit corepressors and associated histone deacetylases and chromatin-modifying enzymes, silencing transcription by socalled active repression (10-12). Ligand binding induces a conformational change in PPAR-RXR complexes, releasing repressors in exchange for coactivators. Ligand-activated complexes recruit the basal transcriptional machinery, resulting in enhanced gene expression. Unlike classical endocrine receptors that bind to highaffinity glandular hormones, PPARs bind to lower-affinity ligands generated from dietary fat or intracellular metabolism. In keeping with their roles as lipid sensors, ligand-activated PPARs turn on feed-forward metabolic cascades to regulate lipid homeostasis via the transcription of genes involved in lipid metabolism, storage, and transport. Additionally, PPARs may suppress inflammation through mechanisms involving the release of antiinflammatory factors or the stabilization of repressive complexes at inflammatory gene promoters $(13,14)$.

Three PPAR isotypes exist in mammals: $\alpha$ (NR1C1), $\gamma$ (NR1C3), and $\delta$ (also known as $\beta$ or NR1C2). PPAR $\alpha$ was the first PPAR to be identified and was demonstrated to be the target of hypolipidemic fibrate drugs and carcinogens that cause peroxisome pro- 
liferation in rodent livers, where PPAR $\alpha$ is abundantly expressed (15). Subsequent cloning efforts identified the $\gamma$ and $\delta$ isotypes. PPAR $\gamma$ is expressed predominantly in adipose tissue, and to a lesser extent in macrophages, muscle, and liver. It has received considerable attention since the mid-1990s, when it was found to be the molecular target of insulin-sensitizing, antidiabetic drugs known as thiazolidinediones $(16,17)$. PPAR $\delta$ remained an enigma for almost a decade after its cloning in 1992 (18-20). Its nearubiquitous tissue expression raised early speculation that it may serve a "general housekeeping role" (20). More recently, receptor knockouts revealed multiple developmental and homeostatic abnormalities in PPAR $\delta$-null mice, including placental defects causing frequent embryonic lethality, decreased adipose mass, myelination defects, altered skin inflammatory responses, and impaired wound healing (21-23). These findings, along with discoveries aided by the development of high-affinity PPAR $\delta$ agonists and additional genetic models, have instead revealed PPAR $\delta$ as a key regulator with the potential to therapeutically target multiple aspects of the metabolic syndrome. This Review will cover the cellular and systemic effects of PPAR $\delta$ action, with special emphasis on its role in the metabolic syndrome.

\section{PPAR $\delta$ ligands}

$\mathrm{X}$-ray crystallography studies of PPAR $\delta$ revealed an exceptionally large ligand-binding pocket of approximately $1,300 \AA^{3}$, similar to that of PPAR $\gamma$ but much larger than the pockets of other nuclear receptors $(24,25)$. The increased dimension is believed to accommodate the binding of various fatty acids or other amphipathic acids to PPARס via hydrogen bonds and hydrophobic interactions (24). Several 14- to 18-carbon saturated fatty acids as well as 16to 20-carbon polyunsaturated fatty acids are suggested to bind PPAR $\delta$ based on ligand screens and competition binding assays, with affinities in the micromolar range $(24,26-28)$. Similar concentrations of synthetic and naturally occurring eicosanoids including prostaglandin $\mathrm{A}_{1}$, iloprost, $15 \mathrm{~d}-\mathrm{J} 2$, and carbaprostacyclin serve as effective PPAR $\delta$ activators (26). Which fatty acids or eicosanoids are physiological ligands of PPAR $\delta$ remains unsettled. However, VLDL particle-derived fatty acids enhance the expression of PPAR $\delta$ target genes in a receptor-dependent manner, suggesting that a variety of VLDL-delivered fatty acids could serve as endogenous receptor agonists (29). Alternatively, combinatorial chemistry and structurebased drug design have facilitated the development of synthetic agonists with nanomolar affinities for PPAR $\delta$, although none is currently marketed for clinical use in humans.

\section{PPAR $\delta$ in lipoprotein metabolism}

A hallmark of the metabolic syndrome is dyslipidemia, marked by elevated triglycerides and low levels of HDL cholesterol. HDL is a driving force in the process of reverse cholesterol transport, reclaiming excess peripheral tissue cholesterol to the liver for excretion. Accordingly, low levels of HDL are associated with an increased risk of coronary artery disease and cardiovascular death in afflicted patients, while overexpression of apoA-I, the major apolipoprotein composing HDL particles, retards atherogenesis in animal models (30-32). Despite clear therapeutic need, currently marketed cholesterol-modifying drugs raise serum HDL levels only modestly.

High-affinity PPAR $\delta$ ligands have revealed an important role for PPAR $\delta$ in lipoprotein metabolism. Treatment of insulin-resistant obese rhesus monkeys with the PPAR $\delta$-selective agonist GW501516 resulted in a dramatic $79 \%$ increase in HDL-C, a $56 \%$ decrease in triglycerides, and a $29 \%$ decrease in LDL cholesterol (33). The profound increase in HDL cholesterol levels correlated with an increase in number, not size, of HDL particles and was accompanied by increased serum levels of the HDL-associated apolipoproteins apoA-I, apoA-II, and apoC-III (33). In addition, fasting insulin levels declined by up to $48 \%$ in the PPAR $\delta$ drug-treated animals (33). Obese and nonobese mice similarly develop an increase of up to $50 \%$ in HDL cholesterol levels when treated with PPAR $\delta$ agonists $(34,35)$. The mechanism by which PPAR $\delta$ activation raises HDL cholesterol levels remains to be elucidated, but studies to date indi-

\section{Table 1}

PPARס gene targets in fat and muscle tissues

Brown fat
Fatty acid transport and oxidation
LCAS (39), VLCAS (39),
ACOX1 (39), m-CPT1 (39),
LCAD (39), VLCAD (39),
HSL (39)
Thermogenesis
UCP1 (39), UCP3 (39)

UCP1 (39), UCP3 (39)

\author{
White fat \\ Thermogenesis \\ UCP1 (39) \\ $\left(\frac{10}{20}\right.$
}

(

\author{
Skeletal muscle \\ Fatty acid transport and oxidation \\ Heart fatty acid-binding protein (48), \\ fatty acid transport protein/CD36 (48), \\ $m$-CPT1 $(40,48)$, PDK4 $(40,48,50)$, \\ HMGCS2 (48), thiolase (48), LCAD (48) \\ Oxidative metabolism \\ Succinate dehydrogenase $(49,60)$, \\ citrate synthase $(49,60)$
}

Mitochondrial respiration and thermogenesis Cytochrome c (40,48), cytochrome oxidase II (40), cytochrome oxidase IV (40), UCP2 $(40,48)$, UCP3 $(40,48)$

Slow-twitch fibers Myoglobin (40), troponin I slow (40)

\section{Cardiac muscle \\ Fatty acid transport and oxidation I-CPT1 (68), m-CPT1 (68), thiolase (68), UCP3 (68), LCAD (68), MCAD (68), PDK4 (68), VLCAD (68), $\operatorname{ACOX1}(68)$}


cate that expression of the reverse cholesterol transporter ABCA1 is enhanced in some tissues upon exposure to PPAR $\delta$ agonists, including human and mouse macrophages as well as human intestinal cells and fibroblasts $(33,35)$. Additional work suggests that PPAR $\delta$ activation reduces intestinal cholesterol absorption via downregulation of the Niemann-Pick C1-like 1 gene (NPC1L1) (35). NPC1L1 is a key mediator of intestinal cholesterol absorption and a putative target for the clinically used cholesterol absorption inhibitor ezetimibe. In light of these findings, PPAR $\delta$ drugs are now in clinical trial for the treatment of human dyslipidemia.

\section{PPAR $\delta$ action in adipose tissue}

Once viewed as a bland storage depot, adipose tissue has emerged as a dynamic endocrine organ (7). Adiposity correlates with insulin resistance and is believed by some to be of primacy in the metabolic syndrome (36). Even mild weight loss may improve serum lipid profiles, glycemic control, and hypertension, yet currently available weight-loss drugs are of limited effectiveness $(37,38)$.

Genetic models and ligand treatment studies have uncovered powerful regulatory functions for PPAR $\delta$ in adipose tissue metabolism and weight control. Using a gain-of-function strategy, transgenic mice encoding a constitutively active viral protein $16-$ PPAR $\delta$ fusion (VP16-PPAR $\delta$ ) expressed under the enhancer-promoter region of the adipose tissue-specific adipocyte fatty acid-binding protein (aP2) gene were developed (39). On a standard chow diet, these fat-specific VP16-PPAR $\delta$ mice had 20\% reduced body weights, $40 \%$ reduced inguinal fat pad masses, decreased adipocyte triglyceride accumulation, and reduced circulating FFAs and triglycerides compared to that of control littermates on the same diet (39). Moreover, in the context of high-fat diet or genetically predisposed obesity, fat-specific VP16-PPAR $\delta$ expression protected against weight gain, adipocyte hypertrophy, hypertriglyceridemia, and steatosis (39). Transcriptional analysis of brown fat in fat-specific VP16-PPAR $\delta$ mice revealed upregulation of genes involved in triglyceride hydrolysis (hormone-sensitive lipase), fatty acid oxidation (long-chain acyl-CoA synthetase, very-long-chain acyl-CoA synthetase, acyl-CoA oxidase), and uncoupling of oxidative phosphorylation (uncoupling protein-1 and -3) (39). Uncoupling protein-1 (UCP1) expression was likewise elevated in white adipose tissue (Table 1). Conversely, PPAR $\delta$-null mice are more susceptible to weight gain and have blunted expression of brown fat UCP1 on a high-fat diet (39). These genetic models collectively suggest that activation of PPAR $\delta$ protects against obesity.

Importantly, PPAR $\delta$ ligands mimic the effects of expression of a constitutively active PPAR $\delta$ transgene. Administration of the synthetic PPAR $\delta$ agonist GW501516 to genetically obese $(d b / d b)$ mice reduced intracellular triglyceride accumulation in the brown fat and liver, analogous to the effects of VP16-PPAR (39). Moreover, PPAR $\delta$ agonists enhanced $\beta$-oxidation in 3T3-L1 preadipocytes by $50 \%$ (39). Most importantly, PPAR $\delta$ ligands retard weight gain in models of high-fat diet-induced obesity $(39,40)$. These results suggest that PPAR synthetic drugs may be therapeutic as antiobesity agents. Short-term (4-month) treatment of obese rhesus monkeys with variable doses of GW501516 did not affect body weight, however, so it remains to be determined whether longterm administration of PPAR $\delta$ drugs will control body weight in monkeys and humans (33).

\section{PPAR $\delta$ action in skeletal muscle}

Skeletal muscle is a key metabolic tissue, accounting for approximately $80 \%$ of insulin-stimulated glucose uptake. It is composed of heterogeneous myofibers that differ in their metabolic and contractile properties, including oxidative slow-twitch (type I), mixed oxidative/glycolytic fast-twitch (type IIA), and glycolytic fast-twitch (type IIB) forms (41). Oxidative myofibers preferentially express enzymes that oxidize fatty acids and contain slow isoforms of contractile proteins, whereas glycolytic myofibers predominantly metabolize glucose and are composed of fast contractile protein isoforms $(41,42)$. Skeletal muscle is highly plastic, adapting to environmental challenges by regulating the composition of slow- and fast-twitch myofibers. Interventions including endurance exercise, physical inactivity, and metabolic diseases such as type 2 diabetes mellitus can induce the trans-differentiation of myofibers (43). This process involves changes in the expression of metabolic and contractile proteins within the myofiber and is influenced by transcription factors including NFAT, FOXO1, and myogenin (43-46).

$P P A R \delta$ is expressed in skeletal muscle at 10- and 50-fold higher levels compared with PPAR $\alpha$ and PPAR $\gamma$, respectively, and it is preferentially found in oxidative rather than glycolytic myofibers $(47,48)$. Consistent with a role for PPAR $\delta$ in the maintenance or formation of oxidative fibers, targeted expression of constitutively active PPAR (VP16-PPAR $\delta$ ) in rodent skeletal muscle increases the proportion of oxidative slow-twitch fibers in predominantly fasttwitch muscle (48). The observed PPAR $\delta$-mediated reprogramming of muscle fiber involves the increased expression of genes related to fatty acid oxidation, mitochondrial respiration, oxidative metabolism, and slow-twitch contractile apparatus (48). Skeletal muscle-specific overexpression of wild-type PPAR $\delta$ induces some features of fiber type remodeling, such as enhanced skeletal muscle oxidative enzyme expression and activity, but fiber type switching is not observed (49). This difference may be due to reduced transcriptional activity of the wild-type PPAR $\delta$ transgene relative to VP16PPAR $\delta$. Indeed, the former presumably requires endogenous receptor ligands, such as fatty acids and prostaglandins, for activation, and these may be present at limiting concentrations or may activate at reduced potency relative to the constitutively active VP16 transactivation domain. PPAR $\delta$-mediated regulation of gene expression has also been confirmed pharmacologically. Treatment of rodents with a synthetic PPAR $\delta$ agonist increases the expression of all of the aforementioned classes of skeletal muscle genes, including those involved in fatty acid oxidation, mitochondrial respiration, oxidative metabolism, and slow-twitch contractile apparatus (40, 48). Similarly, activation of PPAR $\delta$ in cultured skeletal muscle L6 myotubes and $\mathrm{C} 2 \mathrm{C} 12$ cells increases the expression of genes related to oxidative metabolism $(40,50,51)$. A complete roster of genes regulated by PPAR $\delta$ in skeletal muscle is provided in Table 1.

PPAR $\delta$ 's regulation of metabolic and fiber type status has several physiological implications. First, the presence of an increased proportion of oxidative slow-twitch fibers is predicted to decrease skeletal muscle fatigability. For example, increased endurance in marathon runners is linked to a higher proportion of oxidative slow-twitch fibers in their skeletal muscles. Mice with musclespecific VP16-PPAR $\delta$ transgenes have strikingly higher treadmill endurance capacity, running twice as long and far as wild-type mice (48). Second, oxidative fibers have a tremendous impact on fatty acid homeostasis. Both obesity and insulin resistance are linked to a decrease in the proportion of oxidative slow-twitch fibers in skeletal muscle (52-56). Muscle-specific VP16-PPAR $\delta$ transgenic mice, which have a higher proportion of oxidative slowtwitch fibers, are resistant to high-fat diet-induced obesity (48). Activation of PPAR $\delta$ during high-fat feeding increases disposal of 
lipid in skeletal muscles, preventing the storage of excess fat in adipocytes and weight gain $(39,40,49)$. This metabolic remodeling of skeletal muscle may also be responsible for the insulin-sensitizing effects of PPAR $\delta$ agonists in high-fat diet-induced and genetic models of obesity $(39,40)$. Interestingly, in vitro studies also show that PPAR $\delta$ agonist treatment of cultured human skeletal muscle increases insulin-independent glucose uptake (57).

Given the important role of PPAR in skeletal muscle, regulation of its activity by pre- and posttranslational modification is physiologically relevant. Luquet et al. demonstrated that endurance training with 3 weeks of swimming increases the expression of PPAR $\delta$ mRNA and protein in rodent skeletal muscle (49). In addition, a single exhaustive bout of cycling increases PPAR $\delta$ mRNA and protein expression within 3 hours after completion of exercise in humans $(58,59)$. Fasting also increases PPAR $\delta$ expression in skeletal muscle, and it is proposed that PPAR $\delta$ mediates the fasting-dependent rise in skeletal muscle fatty acid oxidation (60). To our knowledge, changes in the expression of muscle PPAR $\delta$ during instances of positive energy balance, such as obesity, have not been measured. In addition to pretranslational modification, muscle PPAR $\delta$ protein may be subject to posttranslational modifications, as multiple intracellular signaling molecules such as protein kinase A and MAPK phosphorylate PPAR $\delta$ and increase its transcriptional activity in vitro (61-63). Like PPARס, MAPK, calcium/calmodulin-dependent kinase, and adenosine monophosphate-activated protein kinase are each activated by exercise in skeletal muscle, and future work will be important to determine interconnections between these factors (64-66).

\section{PPAR $\delta$ action in cardiac muscle}

Fatty acid oxidation is the primary source of energy in the postnatal heart (67). Impaired fatty acid oxidation and a shift to reliance on glucose metabolism are hallmarks of myocardial diseases such as cardiac hypertrophy and congestive heart failure (67). As in skeletal muscle, PPAR $\delta$ is a critical regulator of fatty acid oxidation in cardiac tissue. Cheng et al. showed that cardiac-specific deletion of PPAR $\delta$ suppresses the expression of oxidative genes (68). This leads to impaired fatty acid oxidation and a reciprocal increase in glucose oxidation, along with fat accumulation in cardiomyocytes (68). Moreover, PPAR $\delta$-selective agonists increase fatty acid oxidation via the induction of oxidative genes in isolated neonatal as well as adult rat cardiomyocytes (69) (Table 1). The PPARddependent maintenance of basal fatty acid oxidation is crucial for normal cardiac mechanics. PPAR $\delta$-null hearts are characterized by decreased rates of contraction and relaxation, increased left ventricular end-diastolic pressure, and decreased cardiac output, factors associated with the onset of cardiac failure (68). Indeed, mice with cardiac-specific deletion of PPAR develop age-dependent cardiac lipotoxicity, cardiac hypertrophy, end-stage dilated cardiomyopathy, and decreased survival (68). The protective role of PPAR $\delta$ in the heart has been confirmed by in vitro studies showing that PPAR $\delta$ agonists attenuate phenylephrine-induced cardiac hypertrophy. While phenylephrine suppresses fatty acid oxidation in cardiomyocytes, concomitant activation of PPAR $\delta$ reverses these effects (70). Although PPAR $\delta$ may directly increase the transcription of fatty acid oxidative genes, at least 1 study suggests that effects could also be indirect. Planavila and colleagues showed

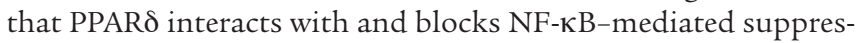
sion of fatty acid oxidation in cardiomyocytes (71). PPAR $\delta$-dependent antagonism of NF- $\mathrm{KB}$ could be particularly important during sepsis, when endotoxins decrease cardiac fatty acid oxidation and initiate cardiac failure $(71,72)$.

\section{PPAR $\delta$ action in macrophage biology and atherosclerosis}

Atherosclerosis is a chronic inflammatory process within the arterial wall that results from the interaction between modified lipoproteins, macrophages, T cells, ECs, and VSMCs (8). It is a major source of morbidity and mortality in the Western world, particularly for patients with the metabolic syndrome. Numerous studies have identified roles for the nuclear receptors PPAR $\gamma$ and liver $\mathrm{X}$ receptor- $\alpha / \beta$ in macrophage cholesterol homeostasis, inflammatory signaling, and atherosclerosis. Like these related receptors, PPAR $\delta$ is expressed by macrophages, in which its functions and implications for atherosclerosis have been studied.

Influence of PPAR on macrophage cholesterol homeostasis. Macrophage cholesterol homeostasis is influenced by PPAR $\gamma$ and the liver $\mathrm{X}$ receptors, but whether PPAR $\delta$ plays a role remains controversial. Oliver et al. showed that treatment of THP-1 human monocytes with the high-affinity agonist GW501516 increased the expression of $A B C A 1$, a critical reverse cholesterol transporter, and doubled apoA-I-specific cholesterol efflux (33). Opposing these results, Vosper et al. found that treatment with a different PPAR $\delta$ drug promoted lipid accumulation in human macrophages exposed to oxidized LDL or THP-1 cells exposed to serum (73). Although PPAR $\delta$ ligand treatment increased apoA-I-specific cholesterol efflux, as reported by Oliver et al., total efflux was diminished (73). ABCA1 was induced, but so too were the cholesterol uptake receptors CD36 and SR-A and the lipid storage-related genes AFABP (aP2) and adipophilin (73). Moreover, the cholesterol efflux gene apoE was repressed (73). These aggregate effects were presumed to contribute to lipid accrual in human macrophages. Alternatively, Lee et al. reported that neither genetic loss of PPAR $\delta$ nor treatment with the PPAR $\delta$ agonist GW501516 influenced cholesterol efflux or accumulation in murine macrophages (13). Li et al. similarly demonstrated no overall effect of the PPAR $\delta$ agonist GW0742 on mouse macrophage cholesterol accumulation, uptake, or apoA-I-dependent efflux, although slightly enhanced cholesterol degradation was observed (74). Taken together, these data suggest that PPAR $\delta$ does not significantly affect macrophage cholesterol metabolism in the mouse, but additional work to decipher a potential contribution of PPAR $\delta$ to human macrophage cholesterol metabolism is needed given the mixed reports described to date.

Role of PPAR $\delta$ in atherogenic inflammation. An in vivo role for PPAR $\delta$ in atherosclerosis was first described using a genetic loss-of-function approach (13). Atherosclerosis-prone LDL receptor-null mice were transplanted with PPAR $\delta$-deficient bone marrow and fed a high-cholesterol diet. After 8 weeks, vascular lesions in PPAR $\delta$ null recipients were at least $50 \%$ smaller than those of wild-type recipient controls (13). There were no differences in cholesterol levels between experimental groups (13). However, PPAR $\delta$-null macrophages expressed decreased levels of inflammatory mediators including monocyte chemoattractant protein-1 (MCP-1), IL-1 $\beta$, and $M M P-9$, while macrophages overexpressing PPAR $\delta$ produced increased levels of inflammatory markers (13). Notably, PPAR $\delta$ ligands inhibited inflammatory gene expression in wild-type macrophages, mimicking the suppressed inflammation elicited in PPAR $\delta$-null cells. Binding studies identified an interaction between PPAR $\delta$ and the inflammatory suppressor protein B cell lymphoma- 6 (BCL-6), which is released from PPAR $\delta$ in a ligand-dependent man- 


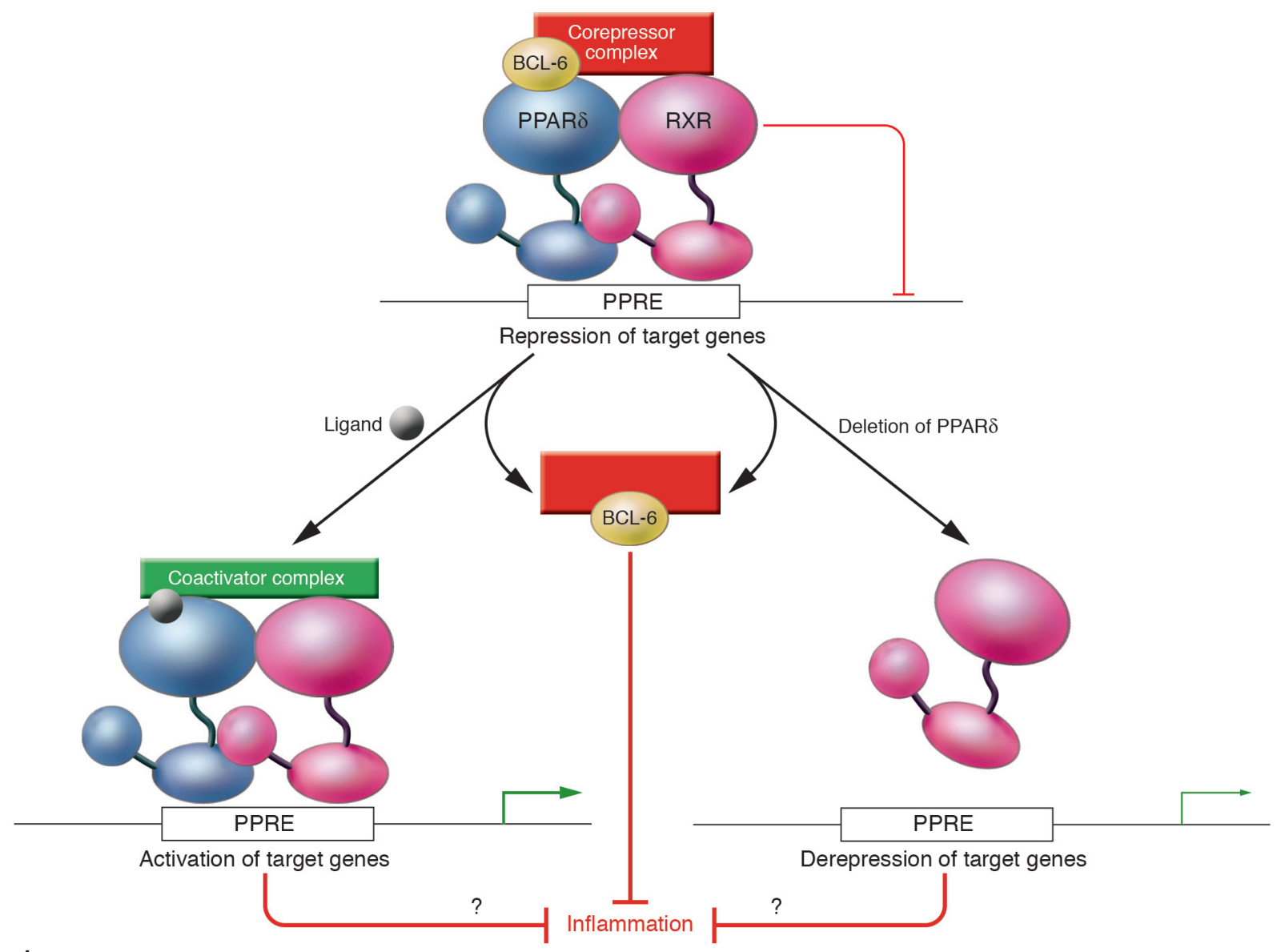

Figure 1

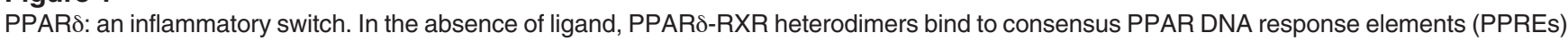
and repress target gene expression by recruiting corepressors and associated repressive proteins including B cell lymphoma-6 (BCL-6) (top). Upon addition of PPAR ligand (bottom left), PPAR $\delta$-RXR heterodimers undergo a conformational shift. This dismisses the corepressor complex, including BCL-6, in exchange for a complex of coactivator proteins and results in enhanced PPAR $\delta$ target gene expression. BCL-6, an inflammatory suppressor protein, is thereby liberated to repress inflammatory gene expression. Genetic deletion of PPAR also releases BCL- 6 and repressor complexes from PPAR $\delta$ target gene promoters, rendering BCL-6 available to suppress inflammation (bottom right). It is unknown whether the expression of direct PPAR $\delta$ target genes (bottom left and right) has antiinflammatory effects. Target gene expression may occur either by ligand-induced transcriptional activation (large green arrow) or more modestly by transcriptional derepression (small green arrow).

ner (13). Thus, genetic loss of PPAR $\delta$ or the addition of PPAR $\delta$ ligands frees a negative regulator of inflammation within the macrophage (13). This "inflammatory switch" model predicts that PPAR ligands, as with genetic deletion of PPAR $\delta$, should ameliorate inflammation (Figure 1) (13). Whether direct genetic targets of PPAR $\delta$ have antiinflammatory effects is unknown (Figure 1). Additional in vitro studies confirm that PPAR $\delta$ ligands are antiinflammatory, suppressing the expression of LPS-induced proinflammatory genes, including iNOS and $\operatorname{COX} 2(13,75)$.

PPAR ligands as therapentics for atherosclerosis. Whether PPAR $\delta$ ligands are therapeutic for atherosclerosis remains controversial; ligand studies have yielded mixed results. To date, 2 published reports have examined the effects of PPAR $\delta$ agonists in mouse models of atherosclerosis. In a study by $L i$ et al., male $L D L R^{-/-}$mice fed an atherogenic diet (containing 1.25\% cholesterol) and treated with $5 \mathrm{mg} / \mathrm{kg} / \mathrm{d}$ of the high-affinity PPAR $\delta$ agonist GW0742 for 14 weeks had no significant changes in atherosclerotic lesion size. Despite this, serum triglyceride levels and vascular wall expression of inflammatory mediators including IFN- $\gamma, T N F-\alpha, M C P-1$,
$I L-1 \beta, V C A M-1$, and ICAM- 1 were significantly diminished in drugexposed animals (74). In contrast, Graham et al. reported that 16-week administration of GW0742 (6 or $60 \mathrm{mg} / \mathrm{kg} / \mathrm{d}$ ) to female $L D L R^{-/-}$mice given an atherogenic diet (containing $0.25 \%$ cholesterol) inhibited atherosclerosis by approximately $30 \%$ (76). VLDL levels were significantly reduced in mice treated with lower doses of PPAR $\delta$ agonist, but these changes were not recapitulated with the higher-dose regimen, and no other changes in serum lipid fractions were identified (76). As in the previously described study, Graham et al. found reduced expression of inflammatory genes including $M C P-1, T N F-\alpha$, and ICAM- 1 within the aortae of PPAR $\delta$ drug-treated mice (76). Serum levels of proinflammatory mediators including MCP-1, RANTES, IL-12, and soluble TNF-R1 were also suppressed in high-dose drug-treated animals (76). Compared with the study by Li et al., the efficacy achieved by Graham et al. could be due to use of higher drug doses, longer treatment duration, lower cholesterol supplementation, or female mice. It is possible that the antiatherosclerotic effects of PPAR $\delta$ compounds require high doses, are more pronounced in advanced lesions or in the setting of modest 


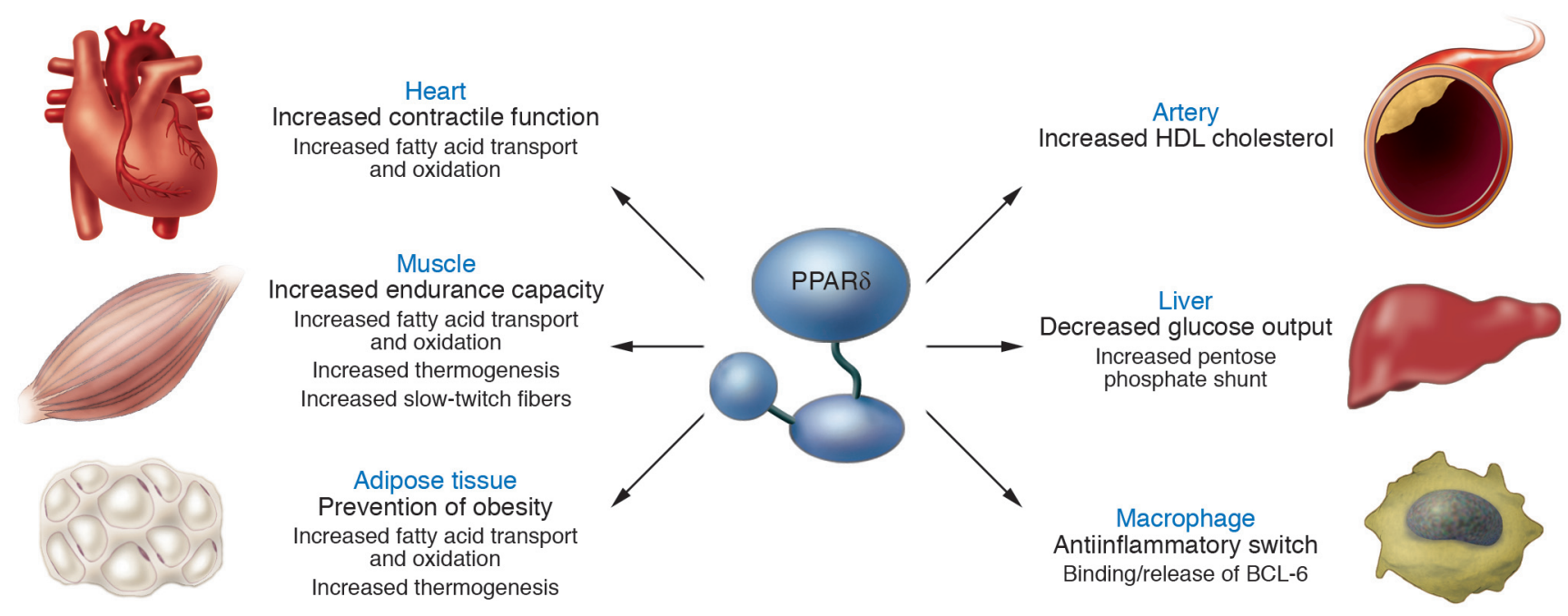

Figure 2

Therapeutic targets of PPAR in the metabolic syndrome. Receptor activation improves multiple aspects of the metabolic syndrome through tissue- and cell-specific effects. In skeletal muscle, PPAR $\delta$ regulates fatty acid transport and oxidation, thermogenesis, and the formation of slow-twitch muscle fibers, resulting in enhanced endurance performance. It likewise activates fatty acid transport and oxidation as well as thermogenesis in adipose tissue, retarding weight gain. PPAR $\delta$ regulates the availability of BCL-6, an inflammatory suppressor protein released upon ligation of PPARס, thereby functioning as an "antiinflammatory switch" to control macrophage-elicited inflammation and atherogenesis. In the liver, PPAR $\delta$ activation suppresses glucose production by upregulating the pentose phosphate shunt. PPAR $\delta$ activation also improves atherogenic dyslipidemia by raising serum HDL cholesterol levels via unclear mechanisms. Additionally, PPAR $\delta$ activation in the heart enhances contractile function and may improve cardiomyopathy.

cholesterol exposure, or are exerted in a sexually dimorphic manner, accounting for the discrepancy among published studies in the $L D L R^{-1-}$ mouse model. Hence, PPAR $\delta$ ligands have antiinflammatory effects in vivo, but whether this is generally sufficient to inhibit atherogenesis will require additional studies.

\section{Conclusion}

PPAR $\delta$ has emerged as a powerful metabolic regulator in diverse tissues including fat, skeletal muscle, and the heart. Its transcriptional program enhances fatty acid catabolism and energy uncoupling, resulting in decreased triglyceride stores, improved endurance performance, and enhanced cardiac contractility (Table 1), respectively. PPAR $\delta$ receptor activation mitigates macrophage inflammatory responses and modulates lipoprotein metabolism to lower triglycerides and robustly raise HDL cholesterol. Additionally, recent studies reveal that PPAR $\delta$ activation in the liver suppresses hepatic glucose output, contributing to improved glucose homeostasis (77). These aggregate effects suggest that high-affinity PPAR $\delta$ synthetic drugs may uniquely target multiple components of the metabolic syndrome, including obesity, insulin resistance, hyperglycemia, dyslipidemia, and atherosclerosis, or other diseases such as cardiomyopathy (Figure 2).

As with any drug anticipated for chronic use in the treatment of metabolic disease, safety issues about PPAR $\delta$-targeted compounds have been raised. Particular attention has focused on a potential connection to carcinogenesis, since PPAR $\delta$ was reported as a downstream target of the oncogenic Wnt/adenomatous polyposis coli (APC)/ $\beta$-catenin pathway (78). A recent study disputes this connection, and further studies to decipher a role for PPAR $\delta$ in cancer have been inconsistent $(21,79-82)$. Experiments using PPAR $\delta$-null mice demonstrated that PPAR $\delta$ is genetically dispensable for colon polyp formation in both the Apc ${ }^{\text {min }}$ genetic mutant and chemi- cally induced colon cancer mouse models, and colon polyp formation was exacerbated in the genetic absence of PPAR $\delta(21,79$, 80). Alternatively, xenografts of human colon cancer cells showed decreased tumorigenesis in cells somatically deleted of PPAR $\delta$, and treatment of $\mathrm{Apc}^{\mathrm{min}}$ mice with high doses of the PPAR $\delta$ ligand GW501516 yielded increases in the number and size of intestinal polyps $(81,82)$. Whether these ligand effects are truly dependent on PPAR $\delta$ has not been established, and study using a PPAR $\delta$ null background is warranted. It is notable that synthetic PPAR $\gamma$ agonists, which have been extensively used in human subjects, enhance colon polyp formation in the Apc ${ }^{\mathrm{min}}$ model yet have not been demonstrated to cause cancer in humans $(83,84)$. Similarly, PPAR $\alpha$ activators cause hepatocarcinomas in rodents but fail to have such deleterious effects in humans. These findings cast doubt on the predictive power of mouse models to determine the human carcinogenicity of PPAR-targeted drugs. Nevertheless, additional toxicology work will be required before PPAR $\delta$ compounds can be marketed for chronic clinical use.

Despite significant strides in understanding PPAR $\delta$, a number of questions remain. For example, based on primate studies, PPAR $\delta$ agonists may have unprecedented potential to raise low HDL cholesterol levels, a problem not well addressed by currently marketed drugs for lipid disorders (33). How PPAR produces these effects remains unclear, but the development and study of tissue-specific receptor knockouts may yield valuable insight. Indeed, further study of PPAR $\delta$ could lead to important advances in understanding HDL biogenesis. With regard to obesity, the potent ability of PPAR $\delta$ to drive adaptive thermogenesis is clearly protective in rodents, but whether its ability to upregulate metabolism will be effective in humans as in mice will require clinical study. In fact, studies in humans suggest that thermogenesis may be a minor mechanism in the prevention of obesity, and physical 
activity-related energy expenditure may have a dominant impact (85). This raises a parallel question of whether PPAR $\delta$ agonists, as with constitutive genetic activation of PPAR $\delta$ in skeletal muscle, can drive the formation of oxidative myofibers and enhance physical activity endurance. Could PPAR $\delta$ drugs used in combination with exercise be a key strategy to increase physical activity-related energy expenditure and overcome the sedentary lifestyle and poor cardiorespiratory fitness considered by some as identifying features of the metabolic syndrome (86)? Further study to define the potential of PPAR $\delta$-targeted drugs to treat cardiovascular disease, given the mixed available experimental data, is also needed. Finally, PPAR $\delta$ is expressed in the brain, liver, $\beta$ cells, VSMCs, and endothelium, each of which has connections to the metabolic syndrome; yet its functions in each of these tissues and cell types are virtually unexplored $(21,22,87,88)$. Unquestionably, a great deal of research will be needed to understand the tissue-specific effects of this near-ubiquitous receptor and its full potential to impact the metabolic syndrome and other disorders.

1. Flegal, K.M., Carroll, M.D., Ogden, C.L., and Johnson, C.L. 2002. Prevalence and trends in obesity among US adults, 1999-2000. JAMA. 288:1723-1727.

2. Ford, E.S. 2005. Risks for all-cause mortality, cardiovascular disease, and diabetes associated with the metabolic syndrome: a summary of the evidence. Diabetes Care. 28:1769-1778.

3. Olshansky, S.J., et al. 2005. A potential decline in life expectancy in the United States in the 21st century. N. Engl. J. Med. 352:1138-1145.

4. Eckel, R.H., Grundy, S.M., and Zimmet, P.Z. 2005. The metabolic syndrome. Lancet. 365:1415-1428.

5. Grundy, S.M. 2005. Point: the metabolic syndrome still lives. Clin. Chem. 51:1352-1354.

6. Perseghin, G., et al. 1999. Intramyocellular triglyceride content is a determinant of in vivo insulin resistance in humans: a $1 \mathrm{H}-13 \mathrm{C}$ nuclear magnetic resonance spectroscopy assessment in offspring of type 2 diabetic parents. Diabetes. 48:1600-1606.

7. Kershaw, E.E., and Flier, J.S. 2004. Adipose tissue as an endocrine organ. J. Clin. Endocrinol. Metab. 89:2548-2556.

8. Glass, C.K., and Witztum, J.L. 2001. Atherosclerosis: the road ahead. Cell. 104:503-516.

9. Grundy, S.M., et al. 2005. Diagnosis and management of the metabolic syndrome: an American Heart Association/National Heart, Lung, and Blood Institute Scientific Statement: Executive Summary. Circulation. 112:e285-e290.

10. Ordentlich, P., Downes, M., and Evans, R.M. 2001. Corepressors and nuclear hormone receptor function. Curr. Top. Microbiol. Immunol. 254:101-116.

11. Jepsen, K., and Rosenfeld, M.G. 2002. Biological roles and mechanistic actions of co-repressor complexes. J. Cell Sci. 115:689-698.

12. Privalsky, M.L. 2004. The role of corepressors in transcriptional regulation by nuclear hormone receptors. Annu. Rev. Physiol. 66:315-360.

13. Lee, C.H., et al. 2003. Transcriptional repression of atherogenic inflammation: modulation by PPARdelta. Science. 302:453-457.

14. Pascual, G., et al. 2005. A SUMOylation-dependent pathway mediates transrepression of inflammatory response genes by PPAR-gamma. Nature. 437:759-763.

15. Issemann, I., and Green, S. 1990. Activation of a member of the steroid hormone receptor superfamily by peroxisome proliferators. Nature. 347:645-650.

16. Lehmann, J.M., et al. 1995. An antidiabetic thiazolidinedione is a high affinity ligand for peroxisome proliferator-activated receptor gamma (PPAR gamma). J. Biol. Chem. 270:12953-12956.

17. Forman, B.M., et al. 1995. 15-Deoxy-delta 12, 14-

\section{Acknowledgments}

We thank Jamie Simon for graphic artwork and Lita Ong and Elaine Stevens for administrative assistance. R.M. Evans is an Investigator of the Howard Hughes Medical Institute at the Salk Institute for Biological Studies and March of Dimes Chair in Molecular and Developmental Biology. G.D. Barish is supported by an NIH/ National Institute of Diabetes and Digestive and Kidney Diseases Individual National Research Service Award (1F32DK071478). V.A. Narkar is supported by the Howard Hughes Medical Institute. This work was supported by the Howard Hughes Medical Institute, the NIH Nuclear Receptor Signaling Atlas orphan receptor program (U19DK62434), the Hillblom Foundation, and the UCSD Specialized Center of Research on Molecular Medicine and Atherosclerosis (P50HL56989).

Address correspondence to: Ronald M. Evans, Gene Expression Laboratory, 10010 North Torrey Pines Road, La Jolla, California 92037-1099, USA. E-mail: evans@salk.edu. prostaglandin $\mathrm{J} 2$ is a ligand for the adipocyte determination factor PPAR gamma. Cell. 83:803-812.

18. Schmidt, A., et al. 1992. Identification of a new member of the steroid hormone receptor superfamily that is activated by a peroxisome proliferator and fatty acids. Mol. Endocrinol. 6:1634-1641.

19. Dreyer, C., et al. 1992. Control of the peroxisomal beta-oxidation pathway by a novel family of nuclear hormone receptors. Cell. 68:879-887.

20. Kliewer, S.A., et al. 1994. Differential expression and activation of a family of murine peroxisome proliferator-activated receptors. Proc. Natl. Acad. Sci. U. S. A. 91:7355-7359

21. Barak, Y., et al. 2002. Effects of peroxisome proliferator-activated receptor delta on placentation, adiposity, and colorectal cancer. Proc. Natl. Acad. Sci. U. S. A. 99:303-308.

22. Peters, J.M., et al. 2000. Growth, adipose, brain, and skin alterations resulting from targeted disruption of the mouse peroxisome proliferator-activated receptor beta(delta). Mol. Cell. Biol. 20:5119-5128.

23. Tan, N.S., et al. 2001. Critical roles of PPAR beta/ delta in keratinocyte response to inflammation. Genes Dev. 15:3263-3277.

24. Xu, H.E., et al. 1999. Molecular recognition of fatty acids by peroxisome proliferator-activated receptors. Mol. Cell. 3:397-403.

25. Takada, I., et al. 2000. Alteration of a single amino acid in peroxisome proliferator-activated receptoralpha (PPAR alpha) generates a PPAR delta phenotype. Mol. Endocrinol. 14:733-740.

26. Forman, B.M., Chen, J., and Evans, R.M. 1997. Hypolipidemic drugs, polyunsaturated fatty acids, and eicosanoids are ligands for peroxisome proliferator-activated receptors alpha and delta. Proc. Natl. Acad. Sci. U. S. A. 94:4312-4317.

27. Yu, K., et al. 1995. Differential activation of peroxisome proliferator-activated receptors by eicosanoids. J. Biol. Chem. 270:23975-23983.

28. Krey, G., et al. 1997. Fatty acids, eicosanoids, and hypolipidemic agents identified as ligands of peroxisome proliferator-activated receptors by coactivator-dependent receptor ligand assay. Mol. Endocrinol. 11:779-791.

29. Chawla, A., et al. 2003. PPARdelta is a very low-density lipoprotein sensor in macrophages. Proc. Natl. Acad. Sci.U. S. A. 100:1268-1273.

30. Miller, N.E., Thelle, D.S., Forde, O.H., and Mjos, O.D. 1977. The Tromso heart-study. High-density lipoprotein and coronary heart-disease: a prospective case-control study. Lancet. 1:965-968.

31. Wilson, P.W., Abbott, R.D., and Castelli, W.P. 1988. High density lipoprotein cholesterol and mortality. The Framingham Heart Study. Arteriosclerosis.
8:737-741.

32. Benoit, P., et al. 1999. Somatic gene transfer of human ApoA-I inhibits atherosclerosis progression in mouse models. Circulation. 99:105-110.

33. Oliver, W.R., Jr., et al. 2001. A selective peroxisome proliferator-activated receptor delta agonist promotes reverse cholesterol transport. Proc. Natl. Acad. Sci. U. S. A. 98:5306-5311.

34. Leibowitz, M.D., et al. 2000. Activation of PPARdelta alters lipid metabolism in $\mathrm{db} / \mathrm{db}$ mice. FEBS Lett. 473:333-336.

35. van der Veen, J.N., et al. 2005. Reduced cholesterol absorption upon PPARdelta activation coincides with decreased intestinal expression of NPC1L1. J. Lipid Res. 46:526-534.

36. Carr, D.B., et al. 2004. Intra-abdominal fat is a major determinant of the National Cholesterol Education Program Adult Treatment Panel III criteria for the metabolic syndrome. Diabetes. 53:2087-2094.

37. [Anonymous]. 1998. Clinical guidelines on the identification, evaluation, and treatment of overweight and obesity in adults: the evidence report. National Institutes of Health. Obes. Res. 6(Suppl. 2):51S-209S.

38. Yanovski, S.Z., and Yanovski, J.A. 2002. Obesity. N. Engl. J. Med. 346:591-602.

39. Wang, Y.X., et al. 2003. Peroxisome-proliferatoractivated receptor delta activates fat metabolism to prevent obesity. Cell. 113:159-170.

40. Tanaka, T., et al. 2003. Activation of peroxisome proliferator-activated receptor delta induces fatty acid beta-oxidation in skeletal muscle and attenuates metabolic syndrome. Proc. Natl. Acad. Sci. U. S. A. 100:15924-15929.

41. Pette, D., and Staron, R.S. 2000. Myosin isoforms, muscle fiber types, and transitions. Microsc. Res. Tech. 50:500-509.

42. He, J., Watkins, S., and Kelley, D.E. 2001. Skeletal muscle lipid content and oxidative enzyme activity in relation to muscle fiber type in type 2 diabetes and obesity. Diabetes. 50:817-823.

43. Mercier, J., Perez-Martin, A., Bigard, X., and Ventura, R. 1999. Muscle plasticity and metabolism: effects of exercise and chronic diseases. Mol. Aspects Med. 20:319-373.

44. McCullagh, K.J., et al. 2004. NFAT is a nerve activity sensor in skeletal muscle and controls activitydependent myosin switching. Proc. Natl. Acad. Sci. U. S. A. 101:10590-10595.

45. Kamei, Y., et al. 2004. Skeletal muscle FOXO1 (FKHR) transgenic mice have less skeletal muscle mass, down-regulated Type I (slow twitch/red muscle) fiber genes, and impaired glycemic control. J. Biol. Chem. 279:41114-41123. 
46. Hughes, S.M., Chi, M.M., Lowry, O.H., and Gundersen, K. 1999. Myogenin induces a shift of enzyme activity from glycolytic to oxidative metabolism in muscles of transgenic mice. J. Cell Biol. 145:633-642.

47. Braissant, O., Foufelle, F., Scotto, C., Dauca, M., and Wahli, W. 1996. Differential expression of peroxisome proliferator-activated receptors (PPARs): tissue distribution of PPAR-alpha, -beta, and -gamma in the adult rat. Endocrinology. 137:354-366.

48. Wang, Y.X., et al. 2004. Regulation of muscle fiber type and running endurance by PPARdelta. PLoS Biol. 2:e294.

49. Luquet, S., et al. 2003. Peroxisome proliferator-activated receptor delta controls muscle development and oxidative capability. FASEB J. 17:2299-2301.

50. Abbot, E.L., et al. 2005. Diverging regulation of pyruvate dehydrogenase kinase isoform gene expression in cultured human muscle cells. FEBS J. 272:3004-3014.

51. Solanes, G., Pedraza, N., Iglesias, R., Giralt, M., and Villarroya, F. 2003. Functional relationship between MyoD and peroxisome proliferator-activated receptor-dependent regulatory pathways in the control of the human uncoupling protein-3 gene transcription. Mol. Endocrinol. 17:1944-1958.

52. Hickey, M.S., et al. 1995. Skeletal muscle fiber composition is related to adiposity and in vitro glucose transport rate in humans. Am. J. Physiol. 268:E453-E457.

53. Higashiura, K., et al. 1999. Alteration of muscle fiber composition linking to insulin resistance and hypertension in fructose-fed rats. Am. J. Hypertens. 12:596-602.

54. Kriketos, A.D., et al. 1996. Interrelationships between muscle morphology, insulin action, and adiposity. Am. J. Physiol. 270:R1332-R1339.

55. Kelley, D.E., Goodpaster, B., Wing, R.R., and Simoneau, J.A. 1999. Skeletal muscle fatty acid metabolism in association with insulin resistance, obesity, and weight loss. Am. J. Physiol. 277:E1130-E1141.

56. Manco, M., et al. 2000. Insulin resistance directly correlates with increased saturated fatty acids in skeletal muscle triglycerides. Metabolism. 49:220-224.

57. Kramer, D.K., et al. 2005. Direct activation of glucose transport in primary human myotubes after activation of peroxisome proliferator-activated receptor delta. Diabetes. 54:1157-1163.

58. Watt, M.J., Southgate, R.J., Holmes, A.G., and Febbraio, M.A. 2004. Suppression of plasma free fatty acids upregulates peroxisome proliferator-activated receptor (PPAR) alpha and delta and PPAR coactivator 1alpha in human skeletal muscle, but not lipid regulatory genes. J. Mol. Endocrinol. 33:533-544.

59. Mahoney, D.J., Parise, G., Melov, S., Safdar, A., and Tarnopolsky, M.A. 2005. Analysis of global mRNA expression in human skeletal muscle dur- ing recovery from endurance exercise. FASEB J. 19:1498-1500.

60. Holst, D., et al. 2003. Nutritional regulation and role of peroxisome proliferator-activated receptor delta in fatty acid catabolism in skeletal muscle. Biochim. Biophys. Acta. 1633:43-50.

61. Lazennec, G., Canaple, L., Saugy, D., and Wahli, W. 2000. Activation of peroxisome proliferator-activated receptors (PPARs) by their ligands and protein kinase A activators. Mol. Endocrinol. 14:1962-1975.

62. Hansen, J.B., et al. 2001. Peroxisome proliferatoractivated receptor delta (PPARdelta)-mediated regulation of preadipocyte proliferation and gene expression is dependent on cAMP signaling. J. Biol. Chem. 276:3175-3182.

63. Gelman, L., Michalik, L., Desvergne, B., and Wahli, W. 2005. Kinase signaling cascades that modulate peroxisome proliferator-activated receptors. Curr. Opin. Cell Biol. 17:216-222.

64. Long, Y.C., Widegren, U., and Zierath, J.R. 2004 Exercise-induced mitogen-activated protein kinase signalling in skeletal muscle. Proc. Nutr. Soc. 63:227-232.

65. Coffey, V.G., et al. 2005. Early signaling responses to divergent exercise stimuli in skeletal muscle from well-trained humans. FASEB J. 20:190-192.

66. Chin, E.R. 2005. Role of Ca2+/calmodulin-dependent kinases in skeletal muscle plasticity. J. Appl. Physiol. 99:414-423.

67. Stanley, W.C., Recchia, F.A., and Lopaschuk, G.D. 2005. Myocardial substrate metabolism in the normal and failing heart. Physiol. Rev. 85:1093-1129.

68. Cheng, L., et al. 2004. Cardiomyocyte-restricted peroxisome proliferator-activated receptor-delta deletion perturbs myocardial fatty acid oxidation and leads to cardiomyopathy. Nat. Med. 10:1245-1250.

69. Cheng, L., et al. 2004. Peroxisome proliferator-activated receptor delta activates fatty acid oxidation in cultured neonatal and adult cardiomyocytes. Biochem. Biophys. Res. Commun. 313:277-286.

70. Planavila, A., et al. 2005. Peroxisome proliferatoractivated receptor beta/delta activation inhibits hypertrophy in neonatal rat cardiomyocytes. Cardiovasc. Res. 65:832-841.

71. Planavila, A., Laguna, J.C., and Vazquez-Carrera, M. 2005. Nuclear factor-kappaB activation leads to down-regulation of fatty acid oxidation during cardiac hypertrophy. J. Biol. Chem. 280:17464-17471.

72. Feingold, K., Kim, M.S., Shigenaga, J., Moser, A., and Grunfeld, C. 2004. Altered expression of nuclear hormone receptors and coactivators in mouse heart during the acute-phase response. $A m$. J. Physiol. Endocrinol. Metab. 286:E201-E207.

73. Vosper, H., et al. 2001. The peroxisome proliferator-activated receptor delta promotes lipid accumulation in human macrophages. J. Biol. Chem.
276:44258-44265.

74. Li, A.C., et al. 2004. Differential inhibition of macrophage foam-cell formation and atherosclerosis in mice by $\operatorname{PPAR} \alpha, \beta / \delta$, and $\gamma$. J. Clin. Invest. 114:1564-1576. doi:10.1172/JCI200418730.

75. Welch, J.S., Ricote, M., Akiyama, T.E., Gonzalez, F.J., and Glass, C.K. 2003. PPARgamma and PPARdelta negatively regulate specific subsets of lipopolysaccharide and IFN-gamma target genes in macrophages. Proc. Natl. Acad. Sci.U. S. A. 100:6712-6717.

76. Graham, T.L., et al. 2005. The PPARdelta agonist GW0742X reduces atherosclerosis in LDLR(-/-) mice. Atherosclerosis. 181:29-37.

77. Lee, C.H., et al. 2006. PPAR delta is a potent insulin sensitizer. Proc. Natl. Acad. Sci. U. S. A. In press.

78. He, T.C., Chan, T.A., Vogelstein, B., and Kinzler, K.W. 1999. PPARdelta is an APC-regulated target of nonsteroidal anti-inflammatory drugs. Cell. 99:335-345.

79. Reed, K.R., et al. 2004. PPARdelta status and Apcmediated tumourigenesis in the mouse intestine. Oncogene. 23:8992-8996.

80. Harman, F.S., et al. 2004. Peroxisome proliferatoractivated receptor-delta attenuates colon carcinogenesis. Nat. Med. 10:481-483.

81. Gupta, R.A., et al. 2004. Activation of nuclear hormone receptor peroxisome proliferator-activated receptor-delta accelerates intestinal adenoma growth. Nat. Med. 10:245-247.

82. Park, B.H., Vogelstein, B., and Kinzler, K.W. 2001. Genetic disruption of PPARdelta decreases the tumorigenicity of human colon cancer cells. Proc. Natl. Acad. Sci. U. S. A. 98:2598-2603.

83. Saez, E., et al. 1998. Activators of the nuclear receptor PPARgamma enhance colon polyp formation. Nat. Med. 4:1058-1061.

84. Lefebvre, A.M., et al. 1998. Activation of the peroxisome proliferator-activated receptor gamma promotes the development of colon tumors in C57BL/ 6J-APCMin/+ mice. Nat. Med. 4:1053-1057.

85. Schoeller, D.A. 2001. The importance of clinical research: the role of thermogenesis in human obesity. Am. J. Clin. Nutr. 73:511-516.

86. Lakka, T.A., et al. 2003. Sedentary lifestyle, poor cardiorespiratory fitness, and the metabolic syndrome. Med. Sci. Sports Exerc. 35:1279-1286.

87. Bishop-Bailey, D., and Hla, T. 1999. Endothelial cell apoptosis induced by the peroxisome proliferatoractivated receptor (PPAR) ligand 15-deoxy-Delta12, 14-prostaglandin J2. J. Biol. Chem. 274:17042-17048.

88. Zhang, J., et al. 2002. Peroxisome proliferator-activated receptor delta is up-regulated during vascular lesion formation and promotes post-confluent cell proliferation in vascular smooth muscle cells. J. Biol. Chem. 277:11505-11512. 